\title{
A Binary Cuckoo Search for Combinatorial Optimization in Multiyear Pavement Maintenance Programs
}

\author{
Feng Xiao $\mathbb{D}^{D}$, Shunxin Yang $\mathbb{D}^{D}$, Jianchuan Cheng, Mingyu Hou $(\mathbb{D}$, and Chenzhu Wang \\ School of Transportation, Southeast University, Nanjing 211189, China \\ Correspondence should be addressed to Shunxin Yang; shunxin.yang@seu.edu.cn
}

Received 11 August 2020; Revised 10 November 2020; Accepted 29 November 2020; Published 22 December 2020

Academic Editor: Annan Zhou

Copyright (c) 2020 Feng Xiao et al. This is an open access article distributed under the Creative Commons Attribution License, which permits unrestricted use, distribution, and reproduction in any medium, provided the original work is properly cited.

\begin{abstract}
For the optimization analysis of pavement maintenance programs, combinatorial optimization is a pervasive problem. Genetic algorithms (GAs) are widely used to solve combinatorial optimization problems in pavement maintenance programs. However, owing to the stochastic search mechanisms underlying GAs, they are more likely to produce a relatively unsatisfactory solution due to premature convergence. Hence, a binary cuckoo search (BCS) algorithm was implemented to solve the optimization problem. To the best of our knowledge, this is the first time that a BCS algorithm has been applied to pavement maintenance management system. Three hypothetical cases are used to investigate and demonstrate the effectiveness of the BCS algorithm, in which uncertainty-based performance degradation is considered. The results of a comparison between GA and BCS clearly justify the advantages of the search paths underlying the BCS in alleviating premature convergence. Therefore, the BCS algorithm can help decision makers to make more appropriate trade-off decisions for pavement maintenance programs.
\end{abstract}

\section{Introduction}

Over the past 20 years, China's highway construction industry has developed rapidly and has now entered the maintenance and management mode. The Department of Transportation in China's Shanxi Province (SXDOT) maintains a total of $5258 \mathrm{~km}$ freeway networks and established a pavement management system (PMS) in collaboration with Southeast University in 2018, so as to achieve the goal of reasonable use of pavement maintenance funds. In the same year, SXDOT and Southeast University collaborated to develop a report on the determination of the threeyear pavement maintenance funds for 2019-2021, in which a genetic algorithm (GA) was used to solve an optimization model, which is essentially a combinatorial optimization problem to gain an optimal fund allocation program. At that time, a few problems, such as a premature one that easily produces a relatively unsatisfactory solution, were identified.

In order to improve the premature problem of GA in PMS, a binary cuckoo search (BCS) algorithm was established to solve combinatorial optimization in pavement maintenance management, according to the theory of cuckoo search (CS). To the best of our knowledge, CS theory has not been applied in the field of pavement maintenance management. Therefore, the current study verifies two objectives: (i) if the BCS algorithm can be used to solve the optimization model in PMS and (ii) if the impact of BCS algorithm is better than GA that is the most widely used method in asset management [1]. To address these two objectives, this study constructed three typical optimization problems that considered the uncertainty of performance degradation and finally applied these two algorithms (BCS and GA) to the three cases. Based on the results, it can be judged if the BCS algorithm is appropriate for solving the optimization model and its effect.

\section{Literature Review}

The problem of finding the best multiyear pavement maintenance and rehabilitation (M\&R) activities can be modeled as a combinatorial optimization problem, where integer variables ( 0 or 1 ) are used to indicate if M\&R activities are carried out on individual pavement sections (facilities) in discrete years [2]. However, because of the huge solution space of the 
combinatorial optimization problem, it is difficult to apply traditional optimization techniques to obtain an optimal $\mathrm{M} \& \mathrm{R}$ program. The study [3] indicated that metaheuristics are effective methods for solving combinatorial optimization problems. In metaheuristics, particle swarm optimization (PSO) [4] and greedy randomized adaptive search procedure algorithms [5] have been used to obtain optimal pavement maintenance programs. In addition, $\mathrm{Li}$ and Sinha [6] developed a heuristic algorithm based on Lagrangian relaxation algorithms to solve the optimization decision model that can be regarded as a multichoice multidimensional knapsack problem in highway asset management. Chu and Huang [7] proposed a solution process that combines Lagrangian relaxation algorithms, greedy algorithms, and off-the-shelf mixed-integer programming solvers to solve the optimal decision-making models of five different strategies.

A GA that is frequently used in pavement management was originally proposed and analyzed by Holland [8]. Due to the mechanics of natural selection and natural genetics, GA is a robust search technique, and it has become more popular in several research fields [9-11]. Chan et al. [12] were the first to introduce GA in pavement maintenance management. Subsequently, GA was used to derive optimal solutions in single-objective optimization $[13,14]$ or all the existing Pareto solutions in multiobjective optimization [15-18].

Based on the network optimization system (NOS) in the Arizona Department of Transportation, Wang et al. [19] developed a GA-based, step method-based, weighted method-based, and constraint method-based NOS and found that the GA-based NOS model is more efficient than the other models because it produces the best objective values. In the assignment of pavement treatment options, Sindi and Agbelie [20] compared GA with mixed-integer programming and discovered that GA is more accurate for the entire network or larger problems. However, there are still some limitations in the application of GA, such as long computing time and premature convergence. Abu-Lebdeh et al. [21] suggested some courses of action to make the application of GAs efficient, rapid, and productive. These include (1) structuring of individuals into subpopulations or various other classes that are treated separately with respect to, for example, application of various operators; (2) division of workload among multiple loosely coupled processors (for example, as in a cluster or network); and (3) hybridizing GAs with other nonevolutionary search methods. Santos et al. [2] proposed a novel adaptive hybrid genetic algorithm (AHGA) that incorporates local search techniques into GA for determining the best pavement $M \& R$ strategy. Thus, the new AHGA is proven to be superior to the traditional GA in terms of efficiency. Tayebi et al. [4] compared GA and PSO in the program of network-level pavement maintenance activities. Using a case study that seeks minimum $M \& R$ costs, the results show that PSO operates faster and more accurately than GA. The assignment of pavement M\&R activities usually includes three types: (1) M\&R cost minimization; (2) pavement performance maximization; and (3) performance maximization and cost minimization, which is a biobjective model. Nevertheless, the authors [4] conducted only the first type of experiment.
The CS algorithm is another metaheuristic algorithm developed by Yang and Suash Deb [22, 23], for solving optimization problems. The CS algorithm had been proved much more efficient in finding the global optima with higher success rates than both GA and PSO [22]. Recent theoretical studies also show that the CS algorithm has global convergence [24].

According to Yang and Suash Deb [23], the CS algorithm is inspired by the reproduction behavior of cuckoos. Cuckoos lay their eggs in the nests of other host birds. While looking for host birds' nests, cuckoos take a path that corresponds to Lévy flight. If a host bird discovers that the eggs are not its own, it either throws these alien eggs away or simply abandons its nest and builds a new nest elsewhere. This process is assumed to find these alien eggs randomly with a certain probability of $P_{a}$. Subsequently, the basic CS algorithm is described using the following three idealized rules:

(1) Each cuckoo lays one egg at a time in a randomly selected nest

(2) The number of host nests is fixed, and the best nests with high-quality eggs will be carried over to the next generations

(3) A host could discover an alien egg with a probability of $P_{a} \in[0,1]$

The original CS algorithm only operates in continuous optimization problems; consequently, to solve the binary discrete optimization problem, a BCS algorithm was developed based on CS theory [25-27]. The BCS algorithm has been successfully applied to complex industrial systems [28] and power systems [29]. However, as mentioned above, it has not been used in the field of pavement maintenance management.

\section{Problem Statement: Combinatorial Optimization in Pavement Maintenance Management}

In pavement maintenance management, engineers must determine which road sections are to be repaired, when repairs should be carried out, and the treatment to be used. As pavement management is still in its infancy in China, engineers are more inclined to choose a specific treatment for a pavement section based on their engineering experience. Therefore, here, the decision-making content for a network-level pavement maintenance program is which road sections to maintain during which year. The treatments are determined according to the decision tree that is established based on engineering experience. In a multiyear pavement maintenance program, the flow of pavement performance changes is shown in Figure 1. Assuming that inspection is performed at the beginning of the first maintenance year, the performance of each pavement sector is known. The M\&R program is performed at a fixed time each year, and the performance value rises immediately after the M\&R activities. Thereafter, following a year of natural deterioration without 


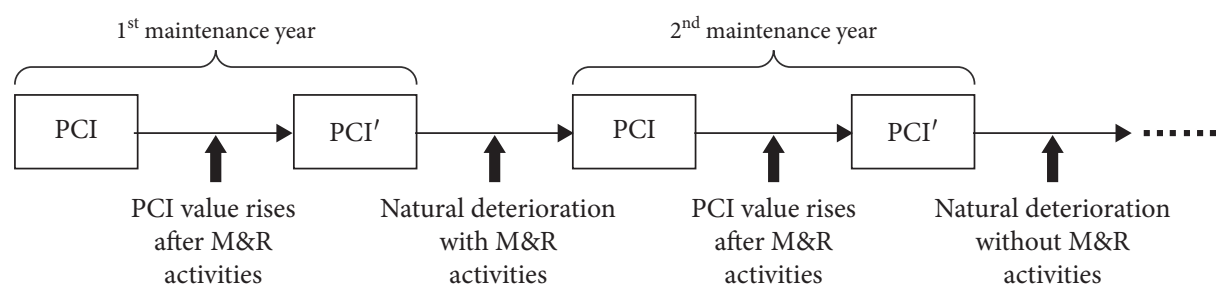

FIgURE 1: Flow of pavement performance changes. $\mathrm{PCI}^{\prime}$ is the PCI value after the M\&R activities.

M\&R activities, the performance value becomes the initial value of the second maintenance year, and so on. The selection of pavement performance indicators followed the existing highway performance assessment standard in China $[30,31]$. In this study, the pavement surface condition index (PCI) in the standard is used as the pavement performance index to reflect the severity of pavement damage. The PCI value range is $0-100$, with 0 indicating extremely poor condition and 100 , a perfect pavement.

Broadly, there are three types of optimization problems: (1) total M\&R cost minimization under a certain pavement performance constraint, (2) pavement performance maximization under a specified budget, and (3) the combination of total M\&R cost minimization and pavement performance maximization. Based on these three types of optimization problems, three case studies are proposed here.

3.1. Cost Minimization. The first case study aims to minimize total M\&R costs over a planning period and meet several practical constraints. It can be formulated as follows:

$$
\operatorname{minimize} Z_{1}=\sum_{t=1}^{T} \sum_{s=1}^{S} \sum_{r=1}^{R}\left(P_{t s r} \times X_{t s} \times L_{s} \times W_{s}\right),
$$

subject to

$$
\begin{aligned}
\mathrm{PCI}_{-} \mathrm{ave}_{t}^{\prime} & \geq \mathrm{PCI}_{t}^{\mathrm{min}}, \quad \forall t=1, \ldots, T, \\
X_{t s} & \in\{0,1\}, \quad \forall t=1, \ldots, T ; s=1, \ldots, S,
\end{aligned}
$$

where

$$
\begin{array}{r}
\mathrm{PCI}_{t s}^{\prime}=\left(1-X_{t s}\right) \times \mathrm{PCI}_{t s}+X_{t s} \times \mathrm{PV}_{r}, \quad \forall t=1, \ldots, T ; \\
s=1, \ldots, S,
\end{array}
$$

$$
\mathrm{PCI}_{(t+1) s}=\mathrm{PCI}_{t s}^{\prime}-\mathrm{PD}_{s r}, \quad \forall t=1, \ldots, T ; s=1, \ldots, S,
$$

$$
\text { PCI_ave }_{t}=\frac{\sum_{s=1}^{S}\left(\mathrm{PCI}_{t s}^{\prime} \times L_{s} \times W_{s}\right)}{\sum_{s=1}^{S}\left(L_{s} \times W_{s}\right)}, \quad \forall t=1, \ldots, T .
$$

3.2. Pavement Performance Maximization. The second case study aims to maximize pavement performance during a planning period, which can be formulated as follows: $\operatorname{maximize} Z_{2}=\frac{\left(\sum_{t=1}^{T}\left(\sum_{s=1}^{s}\left(\mathrm{PCI}_{t s}^{\prime} \times L_{s} \times W_{s}\right) / \sum_{s=1}^{S}\left(L_{s} \times W_{s}\right)\right)\right)}{T}$

subject to

$$
\sum_{s=1}^{S} \sum_{r=1}^{R}\left(P_{t s r} \times X_{t s} \times L_{s} \times W_{s}\right) \geq \text { Budget }_{t}^{\min }, \quad \forall t=1, \ldots, T,
$$

$$
\sum_{t=1}^{T} \sum_{s=1}^{S} \sum_{r=1}^{R}\left(P_{t s r} \times X_{t s} \times L_{s} \times W_{s}\right) \leq \text { Budget }^{\max } .
$$

And Formula (2a) is also subject to formulae (1c), (1d), and (1e).

3.3. Performance Maximization and Cost Minimization. The third case study aims to minimize M\&R costs and maximize performance simultaneously. In this study, both objectives are given a weight and combined into a singleobjective, as shown in formula (3a).

$$
\operatorname{maximize} Z_{3}=w_{1} \times \widetilde{Z}_{1}+w_{2} \times \widetilde{Z}_{2}
$$

subject to formulae (1b)-(1f) and formulae (2b)-(2c), where

$$
\begin{aligned}
& \widetilde{Z}_{1}=\frac{Z_{1}^{\max }-Z_{1}}{Z_{1}^{\max }-Z_{1}^{\min }}, \\
& \widetilde{Z}_{2}=\frac{Z_{2}-Z_{2}^{\min }}{Z_{2}^{\max }-Z_{2}^{\min }} .
\end{aligned}
$$

3.4. Notation List and Explanation of Formulas. All variable explanations in Formulae (1a)-(3c) are listed in Table 1. Formula (1a) expresses the minimization of the total M\&R costs during a planning period. Formula (1b) is a constraint to ensure that the average PCI per year is greater than or equal to $\mathrm{PCI}_{t}^{\mathrm{min}}$. Formula (1c) indicates the range of decision variables and shows that the optimization model is $0-1$ integer programming. Equation (1d) represents the change in PCI value after M\&R activities. Equation (1e) corresponds to a function that calculates PCI before next year's M\&R activities. Equation (1f) is used to calculate the average PCI value after $M \& R$ activities in each year.

The purpose of formula (2a) is to maximize the average PCI after M\&R activities during the planning period. Constraint (2b) ensures that $M \& R$ costs in year $t$ are not 
TABLE 1: Mathematical formulation nomenclature.

\begin{tabular}{|c|c|}
\hline Variable & Description \\
\hline$Z_{1}$ & Total M\&R cost over a planning period \\
\hline$Z_{2}$ & Average value of pavement performance over a planning period \\
\hline$Z_{3}$ & Combined value of performance and M\&R cost \\
\hline$T, t$ & $T$ is length of the planning period; $t$ is the ordinal of $T$ \\
\hline$S, s$ & $S$ is number of pavement sections; $s$ is the ordinal of $S$ \\
\hline$R, r$ & $R$ is number of M\&R activities; $r$ is the ordinal of $R$ \\
\hline$P_{t s r}$ & Price (unit cost) for applying M\&R activity $r$ to pavement section $s$ in year $t$ \\
\hline$X_{t s}$ & Decision variable, equal to 0 if pavement section $s$ do nothing in year $t$, otherwise equal to 1 \\
\hline$L_{s}$ & Length of pavement section $s$ \\
\hline$W_{s}$ & Width of pavement section $s$ \\
\hline PCI_ave ${ }_{t}^{\prime}$ & Average PCI value of road network in year $t$ \\
\hline $\mathrm{PCI}_{t}^{\min }$ & Average PCI minimum value of road network in year $t$ \\
\hline $\mathrm{PCI}_{t s}^{\prime}$ & PCI after M\&R activities for pavement section $s$ in year $t$ \\
\hline $\mathrm{PCI}_{t s}$ & PCI of pavement section $s$ in year $t$ \\
\hline $\mathrm{PV}_{r}$ & Resetting value of pavement performance after obtaining M\&R activity $r$ \\
\hline $\mathrm{PCI}_{(t+1) s}$ & PCI of pavement section $s$ in year $t+1$ \\
\hline $\mathrm{PD}_{s r}$ & Performance degradation of M\&R activity $r$ adopted in previous year for pavement section s. It is an uncertain value \\
\hline Budget $_{t}^{\min }$ & Minimum budget funds for M\&R activities in year $t$ \\
\hline Budget ${ }^{\max }$ & Maximum budget funds for M\&R activities in the planning horizon \\
\hline${\underset{\sim}{1}}_{1}, \underset{w_{2}}{w_{2}}$ & Weights given to total M\&R cost and pavement performance \\
\hline$\widetilde{Z}_{1}, \widetilde{Z}_{2}^{2}$ & Standardized versions of formulas (1a) and (2a) \\
\hline$Z_{1}^{\max }, Z_{1}^{\min }$ & Maximal and minimal possible values of $Z_{1}$ \\
\hline$Z_{2}^{\max }, Z_{2}^{\min }$ & Maximal and minimal possible values of $Z_{2}$ \\
\hline
\end{tabular}

lower than Budget ${ }_{t}^{\min }$. Constraint (2c) ensures that the total cost of $M \& R$ activities are not higher than Budget ${ }^{\max }$. Formula ( $3 \mathrm{a})$ is an objective function to minimize the total $M \& R$ costs and maximize pavement performance simultaneously. Formulas (3b) and (3c) express the standardization of formulas (1a) and (2a), respectively. According to the attributes, these three case studies could be considered as combinatorial optimization.

\section{Binary Cuckoo Search Algorithm Framework}

In this study, the optimization decision model in pavement management is a binary discrete optimization problem; hence, a BCS algorithm was adopted instead of the CS algorithm. The BCS algorithm includes the following important parts: (1) creation of new solutions by Lévy flight, (2) binary variable handling, (3) fitness of solutions, and (4) creation of new solutions by random walk. Figure 2 shows the decision variables (M\&R plan) in the BCS algorithm, the detailed flow of which is given in Figure 3.

4.1. Creation of New Nests by Lévy Flight. When generating new solutions $x_{i}^{(g+1)}$, a Lévy flight is performed:

$$
\begin{aligned}
x_{i}^{(g+1)} & =x_{i}^{(g)}+\alpha \otimes \operatorname{Lévy}(\beta), \\
\operatorname{Lévy}(\beta) & =\frac{u}{|v|^{(1 / \beta)}}, \\
u & \sim N\left(0, \sigma^{2}\right), \\
v & \sim N(0,1), \\
\sigma & =\left\{\frac{\Gamma(1+\beta) \sin ((\pi \beta) / 2)}{\beta \Gamma((1+\beta) / 2) 2^{((\beta-1) / 2)}}\right\}^{(1 / \beta)},
\end{aligned}
$$

where $\alpha>0$ is the step size that should be related to the scales of the problem of interest. In most cases, $\alpha=1$. The product $\otimes$ represents entrywise multiplications. $u$ and $v$ are random numbers subject to a normal distribution. $\Gamma(z)$ is the Gamma function, and $\Gamma(z)=\int_{0}^{+\infty} t^{z-1} e^{-t} \mathrm{~d} t . \beta$ is a constant that is taken as 1.5. Here, the generation of random numbers with Lévy flights is performed by using the Mantegna algorithm [32] for a symmetric Lévy stable distribution [33].

4.2. Binary Variable Handling. There are two different approaches for handling binary variables [27], and one approach was adopted for handling binary variables.

$$
x_{i}^{(g+1)}(\text { new })= \begin{cases}0, & \text { if } x_{i}^{(g+1)} \leq 0, \\ \operatorname{round}\left(x_{i}^{(g+1)}\right), & \text { if } 0<x_{i}^{(g+1)}<1, \\ 1, & \text { otherwise. }\end{cases}
$$

4.3. Fitness of Solutions. The solutions (i.e., nests) were evaluated using a user-defined objective function (or fitness). The three objectives are total $M \& R$ costs in the first case, average PCI after M\&R activities in the second case, and a combination of the first two goals in the third case. Owing to the uncertainty of performance degradation, a stochastic simulation method must be used in the solution of fitness. If a solution violates the constraint, it is discarded by assigning an appropriate penalty value. In the first case, the penalty value is set to a larger value. In the second and third cases, the penalty value is -1 .

4.4. Creation of New Solutions by Random Walk. This step is to update the solutions through a local random walk, as suggested in $[27,34]$, controlled by the switching parameter $P_{a}$. The local random walk can be expressed as 


\begin{tabular}{|c|c|c|c|c|c|c|c|c|c|c|c|c|}
\hline \multicolumn{4}{|c|}{$1^{\text {st }}$ year } & \multicolumn{4}{|c|}{$2^{\text {nd }}$ year } & \multicolumn{4}{|c|}{$3^{\text {rd }}$ year } & \\
\hline $\mathrm{X}_{1}$ & $\mathrm{X}_{2}$ & $\ldots$ & $\mathrm{X}_{\max }$ & $\mathrm{X}_{1}$ & $\mathrm{X}_{2}$ & $\ldots$ & $X_{\max }$ & $\mathrm{X}_{1}$ & $\mathrm{X}_{2}$ & $\ldots$ & $\mathrm{X}_{\max }$ & $\ldots$ \\
\hline 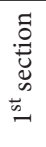 & 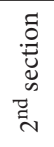 & & 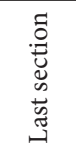 & 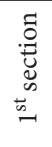 & 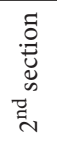 & & 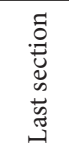 & 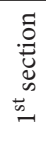 & 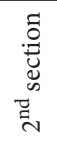 & & 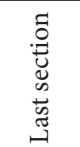 & \\
\hline
\end{tabular}

Figure 2: Representation of decision variables (M\&R plan).

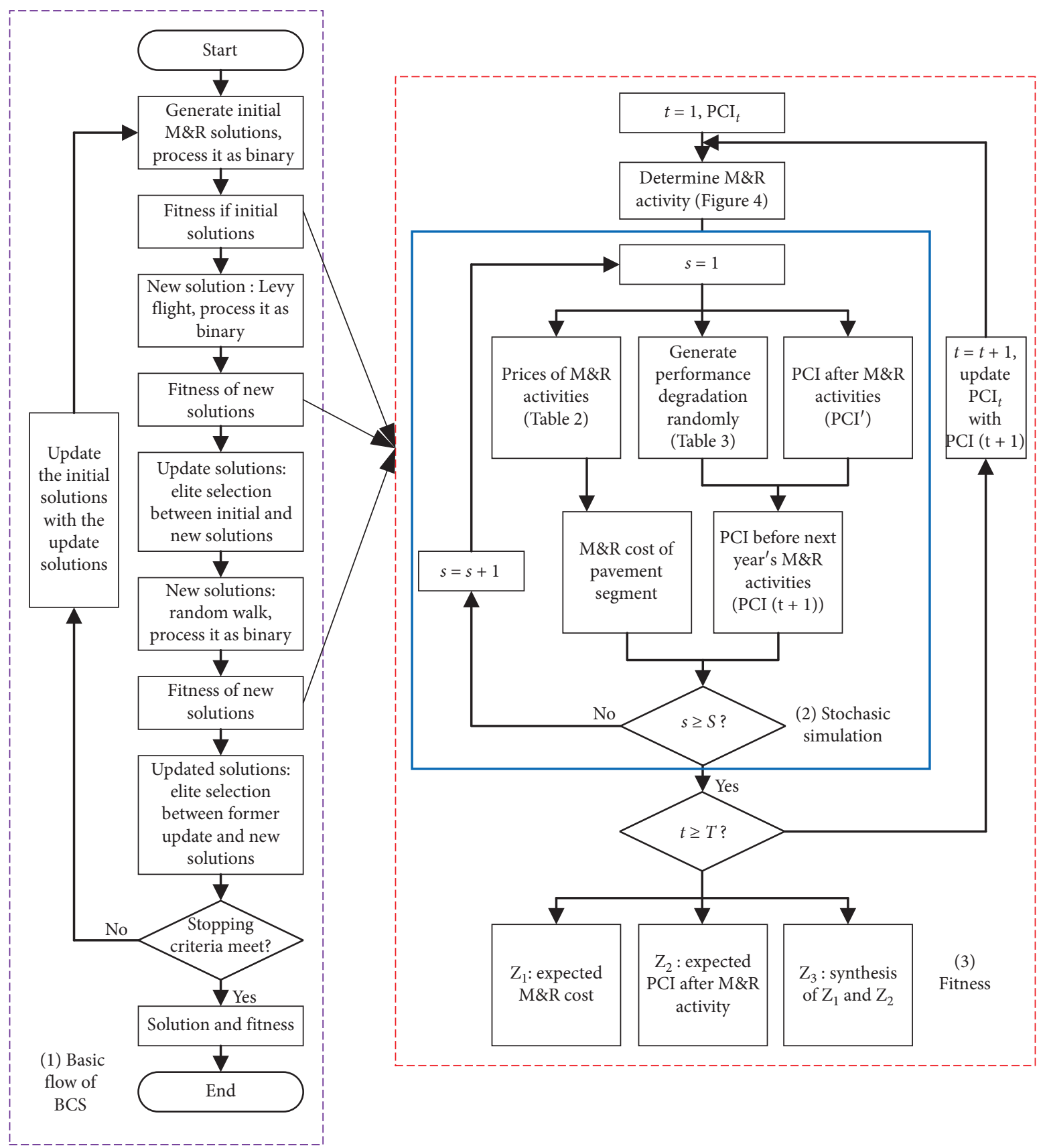

FIGURE 3: Binary cuckoo search (BCS) framework. $S$ represents number of sample points, and $T$ is the planning horizon. 


$$
x_{i}^{(g+1)}=x_{i}^{(g)}+\alpha \otimes H\left(P_{a}-\varepsilon\right) \otimes\left(x_{j}^{(g)}-x_{k}^{(g)}\right),
$$

where $x_{j}^{(g)}$ and $x_{k}^{(g)}$ are two different solutions selected by random permutation at generation $g . \mathrm{H}(z)$ is a Heaviside function. $\varepsilon$ is a random number that is subject to uniform distribution.

\section{Three Cases}

5.1. General Information. To prove the superiority of BCS in solving the combinatorial optimization model in pavement maintenance management, a commonly used GA is used for comparison. This study used a set of actual pavement data (30 pavement sections) from a freeway in Shanxi Province, China. In this case study, the initial PCI value ranged from 80.24 to 100 with an average PCI of 90.1. A three-year M\&R plan (year 2019-2021) was programmed in three examples. In the first example, the average PCI value in each year must be greater than or equal to 92 . In the second example, the road network's annual M\&R costs must not be less than RMB¥ 10,000 (approximately, US\$ 1,421), and total M\&R costs must not exceed RMB¥ 600,000 (approximately, US\$ 85,241).

Figure 4 describes a decision tree for selecting a specific M\&R activity for the pavement section $s$ in year $t$ when $X_{t s}=$ 1 in this study. Each M\&R price is shown in Table 2, where the US dollar exchange rate and China's RMB is set as 1: 7.0389. Notably, preventive maintenance includes fog seal, nova-surfacing, and nova-chip; rehabilitation can be subdivided into medium and heavy rehabilitation. The width of each pavement section was set to $3.8 \mathrm{~m}$. In a maintenance year (refer to Figure 1), if a pavement section receives $M \& R$ activities (excluding doing nothing), its PCI value is reset to $100\left(\mathrm{PCI}^{\prime}=100\right)$; if a pavement section receives doing nothing, its $\mathrm{PCI}$ value remains unchanged $\left(\mathrm{PCI}^{\prime}=\mathrm{PCI}\right)$.

Besides, the uncertainty of pavement performance degradation is also considered. The degradation of pavement sections at different locations after different types of $M \& R$ activities is set to conform to the normal distribution, and the corresponding expected value $(\mu)$ and variance value $(\sigma)$ are shown in Table 3 . In the process of stochastic simulation, the sample size $(S)$ was set to 100 (Figure 3).

5.2. Selection of BCS and GA Parameters. The selection operator in GA adopted a similar elite selection method as $\mathrm{BCS}$, and the procedure for both algorithms is controlled to keep it consistent (see the pseudocodes in Tables 4 and 5 in Supplementary Materials). Besides, the common parameter values of both algorithms were set to be the same. For example, the population size is 20 , and the initial solutions are randomly generated. Five groups having different population size were tested in the three examples (see Table 6 in Supplementary Materials), and the results show that BCS could converge to a better target value than GA. This further demonstrates that a population size of 20 will not affect this study conclusion. In the GA method, the crossover probability is 0.9 , and the mutation probability is 0.05 . We tested nine groups with different probabilities of crossover and mutation, and the results are not much different, indicating that different values of crossover and variation within a small range are insensitive to the final conclusion (see Table 7 in Supplementary Materials). In BCS, the discovery rate of alien eggs $\left(P_{a}\right)$ is 0.25 , which has been proved to be insensitive to the convergence rate, so we do not have to calibrate this parameter [23]. The search domain in BCS is between -10 and 10 in the three examples considered. Five groups of different search domains were tested, and the results show that various search domains have little influence on the final conclusion (see Table 8 in Supplementary Materials). The maximum number of generations is 5000, which is enough to make BCS and GA converge to stable optimal values (see Figures 5-7).

5.3. Numerical Results. The goal of the first example is to minimize total $M \& R$ costs while meeting the minimum pavement performance requirements. In Figure 5, the total cost of the recommended M\&R plan obtained from GA finally converged to RMB 323,452 (i.e., approximately, US\$ 45,952), and BCS finally converged to RMB¥ 289,178 (approximately, US\$ 41,082).

In order to evaluate the performance of the two recommended M\&R plans obtained from both GA and BCS in terms of total cost, the pavement performance curve and pavement deterioration curve of both recommended $M \& R$ plans are displayed in Figure 8. The pavement performance curve displays the average PCI value before and after M\&R activities, respectively, for each year of the three-year maintenance period. Under the premise of meeting the minimum performance requirements, the average pavement performance for BCS is lower than that for GA in almost every year during the planning period. This is consistent with the annual budget allocation in Figure 9, where the $M \& R$ cost of BCS in the first two years is much lower than that of GA. The results in Figures 8 and 9 indicate that, under the minimum performance constraint, BCS can find an optimal solution with lower average pavement performance and lower total M\&R costs compared with GA. In other words, in the case of minimizing the total M\&R cost, BCS is better than GA in finding the recommended M\&R plan.

The objective of the second example is to maximize the average PCI after M\&R activities while meeting the $M \& R$ cost requirements. For the recommended M\&R plans solved from GA and BCS, the average PCI after M\&R activities converged to 98.657 and 99.193, respectively (see Figure 6). In order to further investigate why the recommended M\&R plan from BCS has a larger average PCI after M\&R activities, the average pavement performance curves and budget allocation in each year, which are displayed, respectively, in Figures 10 and 11, were investigated.

The average pavement performance curves reveal that the recommended M\&R plan from BCS maintains a higher average PCI after the M\&R activities in 2019 and 2021, which corresponds with the annual budget allocation during the entire planning period. In Figure 11, the budget for the recommended M\&R plan from BCS is significantly higher than that from GA in 2019, and vice versa in 2020. In 2021, 


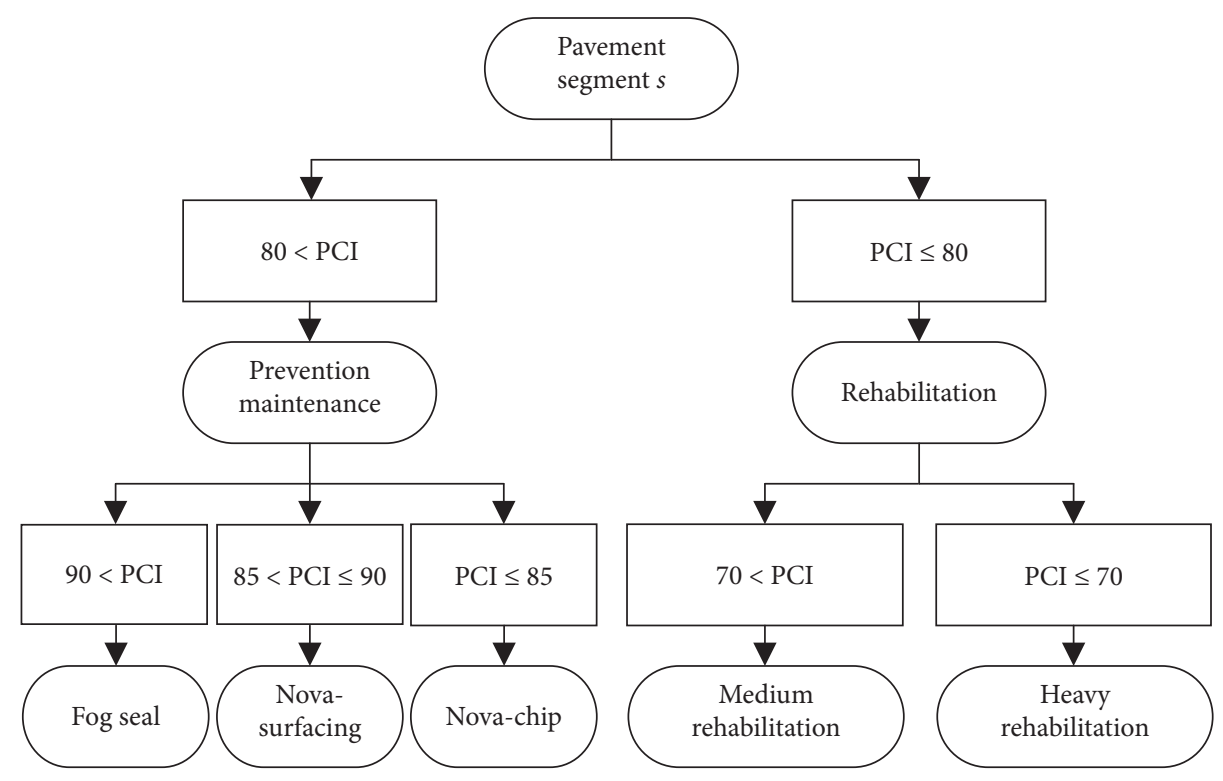

Figure 4: Decision tree.

TABLe 2: Pavement preservation strategy and cost.

\begin{tabular}{|c|c|c|c|}
\hline M\&R types & M\&R activities & Details & Price $/ \mathrm{m}^{2}$ \\
\hline \multirow{3}{*}{$\begin{array}{l}\text { Preventive } \\
\text { maintenance }\end{array}$} & Fog seal & A light application of liquid asphalt emulsion to oxidized pavement surface & $¥ 20(\$ 2.8)$ \\
\hline & Nova-surfacing & $\begin{array}{c}\text { One of the slurry seal that is a mixture of asphalt, aggregate, water, and additives } \\
\text { applied to the pavement surface }\end{array}$ & $¥ 40(\$ 5.7)$ \\
\hline & Nova-chip & $\begin{array}{l}\text { One of the chip seal that is an application of asphalt emulsion and a combination } \\
\text { of asphalt with fine aggregate }\end{array}$ & $¥ 70(\$ 9.9)$ \\
\hline \multirow{2}{*}{ Rehabilitation } & $\begin{array}{l}\text { Medium } \\
\text { rehabilitation }\end{array}$ & Overlay greater than $4 \mathrm{~cm}$ but not more than $10 \mathrm{~cm}$ & $¥ 200(\$ 28.4)$ \\
\hline & $\begin{array}{l}\text { Heavy } \\
\text { rehabilitation }\end{array}$ & Overlay greater than $10 \mathrm{~cm}$ but not more than $18 \mathrm{~cm}$ & $¥ 300(\$ 42.6)$ \\
\hline
\end{tabular}

TABLE 3: Normal distribution parameters of performance degradation in different situations.

\begin{tabular}{|c|c|c|c|}
\hline Section locations & M\&R activities in the previous year & Perfor & dation \\
\hline Pavement sections in tunnels & $\begin{array}{c}\text { Doing nothing } \\
\text { Preventive maintenance } \\
\text { Rehabilitation }\end{array}$ & $\begin{array}{l}\mu=4.89 \\
\mu=3.76 \\
\mu=2.05\end{array}$ & $\sigma=0.1$ \\
\hline Pavement sections in bridges & $\begin{array}{c}\text { Doing nothing } \\
\text { Preventive maintenance } \\
\text { Rehabilitation } \\
\end{array}$ & $\begin{array}{l}\mu=5.21 \\
\mu=3.02 \\
\mu=2.05\end{array}$ & \\
\hline Pavement sections in other road locations & $\begin{array}{c}\text { Doing nothing } \\
\text { Preventive maintenance } \\
\text { Rehabilitation } \\
\end{array}$ & $\begin{array}{l}\mu=4.32 \\
\mu=3.16 \\
\mu=2.87\end{array}$ & \\
\hline
\end{tabular}

the former is slightly higher than the latter. It can be inferred that, compared with GA, the recommended M\&R plan from BCS seems inclined to invest more maintenance funds and improve pavement performance during the early planning period, under the condition of total funding constraints during the entire planning period. This strategy can effectively improve overall pavement performance during a multiyear maintenance period, as confirmed in [35]. The total M\&R cost of these two recommended plans from both BCS and GA is RMB¥ 599,830 (approximately, US\$ 85,216) and RMB¥ 599,137 (approximately, US\$ 85,118), respectively. The results show that, under the constraints of total M\&R fund (RMB $¥ 600,000$ or approximately US\$ 85,241 ), the recommended plan from BCS makes full use of the maintenance fund compared with GA. Therefore, in the case of maximizing average PCI after the M\&R activities during the entire planning period, an effective maintenance strategy and the full use of maintenance funds makes the recommended plan from BCS better than GA.

Unlike the previous two cases, the third example optimizes both pavement performance and total M\&R cost simultaneously. In this study, both weights $\left(w_{1}, w_{2}\right)$ are set to 


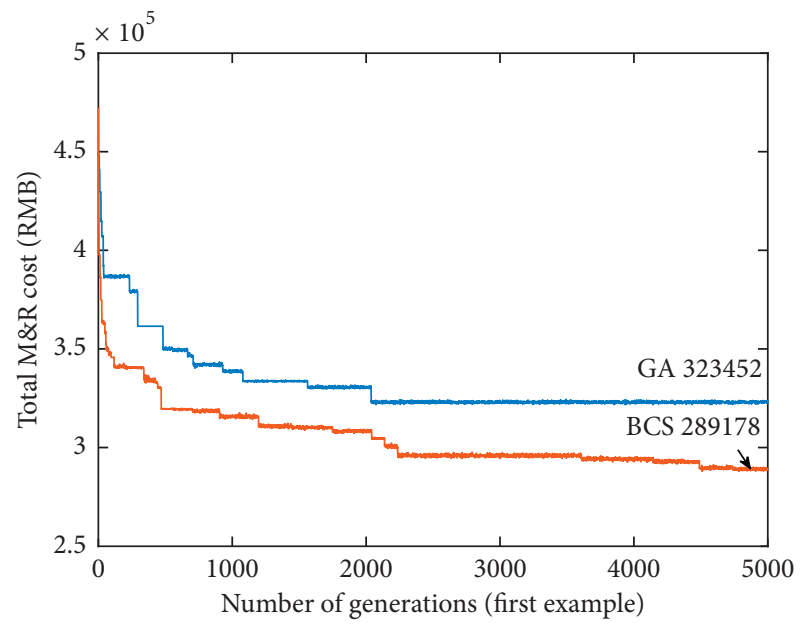

Figure 5: Convergence curve of total cost minimization.

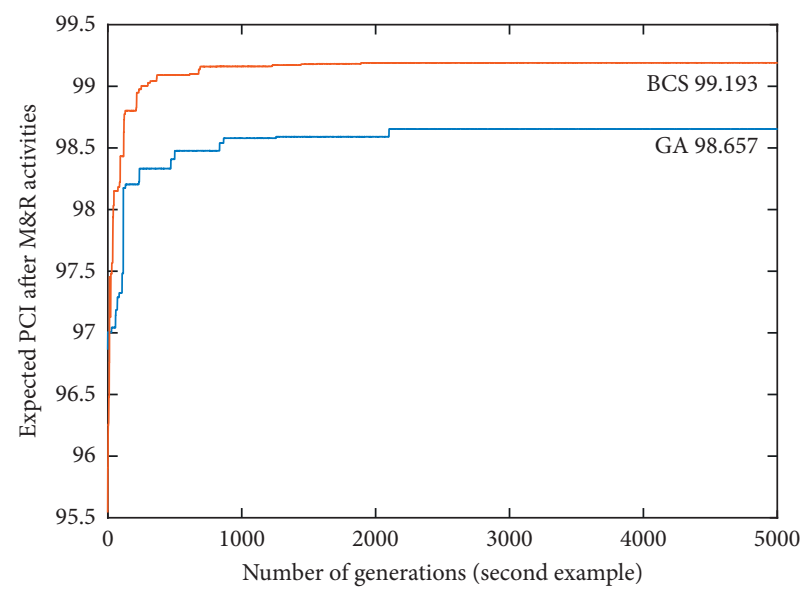

FIGURE 6: Convergence curve of expected PCI after M\&R activities.

0.5 because the importance of these two objectives is considered equally important. Figure 7 displays the convergence curves of these two objectives obtained from GA and BCS. The recommended M\&R plans from both BCS and GA with the objectives of expected PCI after M\&R activities are 96.585 and 96.510 , respectively. The total M\&R cost for these two recommended plans is RMB¥ 358,231 (approximately, US\$ 50,893) and RMB¥ 359,368 (approximately, US\$ $51,055)$, respectively.

In order to evaluate pavement performances and total $M \& R$ costs of the two biobjective $M \& R$ plans, a pavement performance curve and budget allocation were developed for each year in Figures 12 and 13, respectively. These two figures show that, in the first year of the planning period, the recommended M\&R plan from BCS compared to GA maintains a relatively low pavement performance with a relatively small budget investment. This corresponds to the pavement performance curves of the total M\&R cost minimization objective (i.e., the first example). Yet, as a biobjective model, it also attempts to maximize pavement performance. Therefore, in the second year, the strategy of

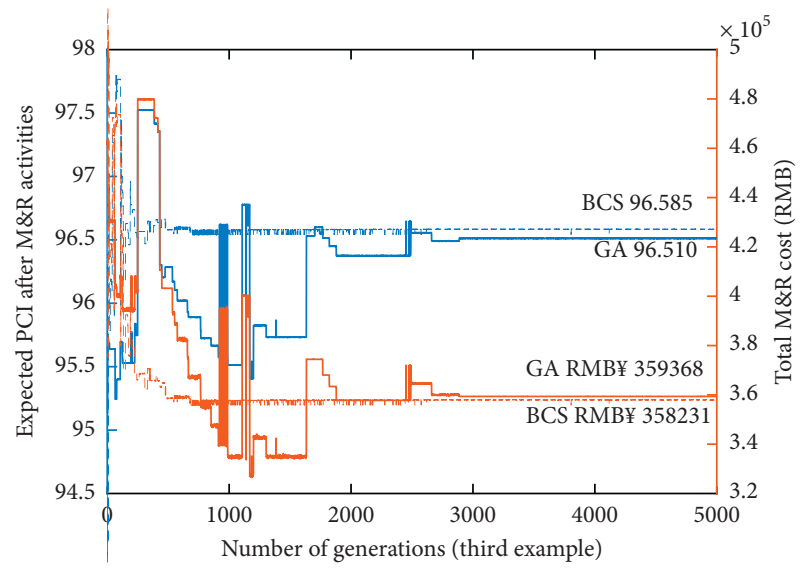

Figure 7: Convergence curve of biobjective model.

the M\&R plan from BCS is to increase budget investment and improve pavement performance compared with the $M \& R$ plan from GA. In the third year, the investment of these two M\&R plans is not significantly different, so the former M\&R plan from BCS continues with the higher pavement performance value of the previous year.

Corresponding to the results of the previous two singleobjective cases, in the biobjective case, the recommended $M \& R$ plan from GA requires larger M\&R costs; however, pavement performance after the $M \& R$ activities is relatively low. Consequently, in the case of optimizing both pavement performance and total M\&R cost simultaneously, BCS is better than GA in exploring an optimal M\&R plan.

\section{Discussion}

For the optimal M\&R plans recommended by both BCS and GA, in the first example of minimizing total M\&R costs, the cost converged to RMB¥ 289,178 (approximately, US\$ 41,082) and RMB¥ 323,452 (approximately, US\$ 45,952), respectively. In the second example of maximizing average PCI after implementing various M\&R activities, the average PCI converged to 99.193 and 98.657 , respectively. In the third example of performance maximization and cost minimization, the average PCI converged to 96.585 and 96.510, and the total M\&R cost converged to RMB¥ 358,231 (approximately, US\$ 50,893) and RMB¥ 359,368 (approximately, US\$ 51,055), respectively. Moreover, we analyzed the reasons why the optimal M\&R plans from BCS were better than those from GA in the above three examples through the average pavement performance curves and budget allocation in each year.

Through an intuitive comparison of the final convergence results of both BCS and GA, it can be concluded that BCS outperforms GA in solving the combinatorial optimization problem in a multiyear pavement maintenance program. Comparing the pseudocode of BCS and GA in Supplementary Materials, the only significant difference between the two is the search paths that follow Lévy flight and random walk for BCS and crossover and mutation for GA. The superior performance of BCS over GA is 


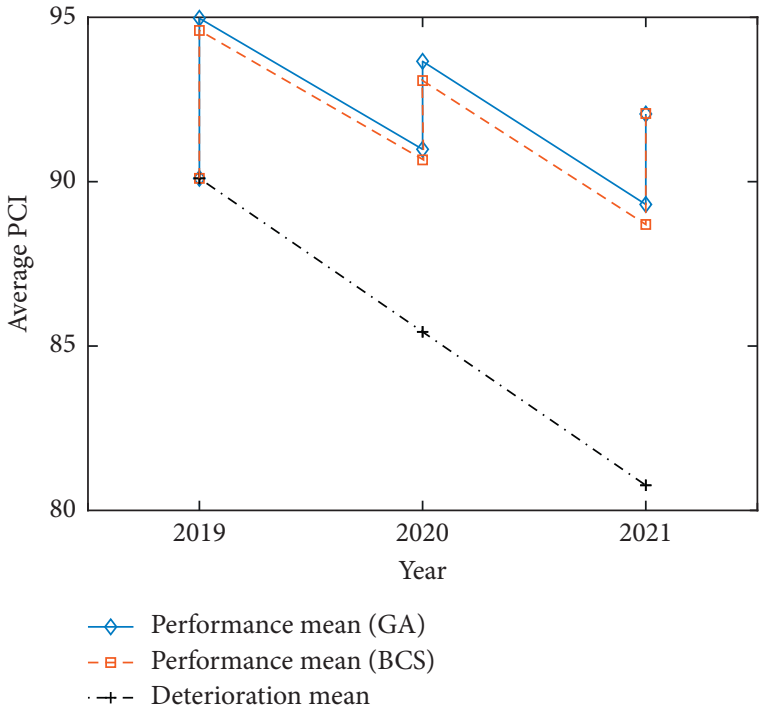

Figure 8: Pavement performance under M\&R cost minimization.

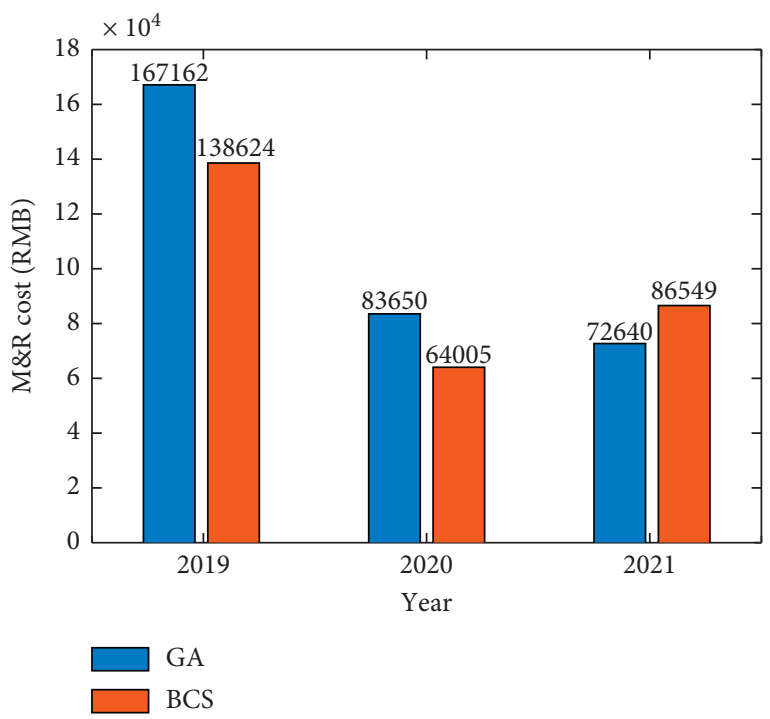

FIGURE 9: Budget allocation in each year under M\&R cost minimization.

attributable partly to the infinite mean and variance of Lévy distribution. A BCS can explore more effectively than GA that uses random search following uniform distribution, especially in the large search space. Coincidentally, most of the combinatorial optimization problems in pavement maintenance management have an extremely large search space.

On the other hand, in the BCS algorithm, the elitist selection operators could ensure that the best solution is passed to the next iteration, so that exploitation around the best solutions is performed by using a random walk:

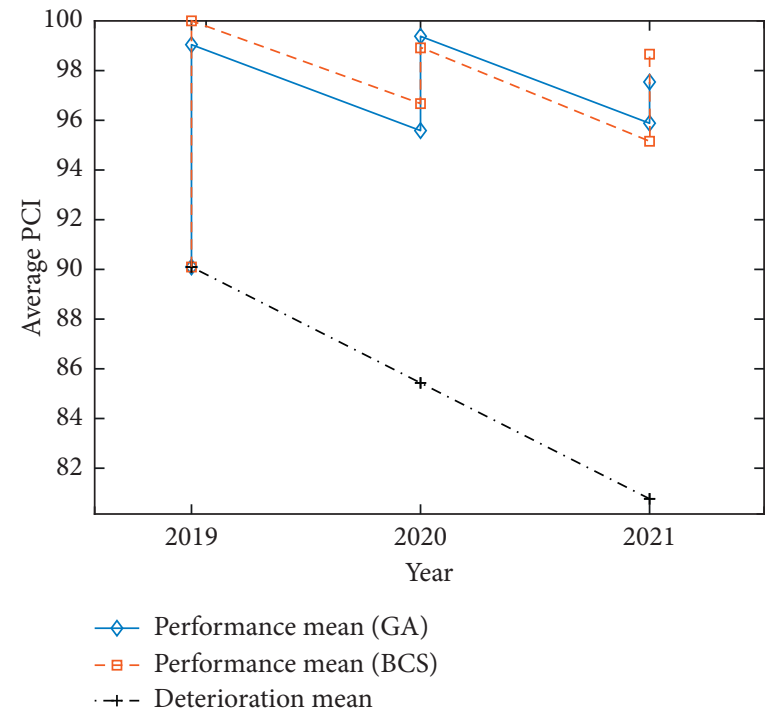

Figure 10: Pavement performance under performance maximization.

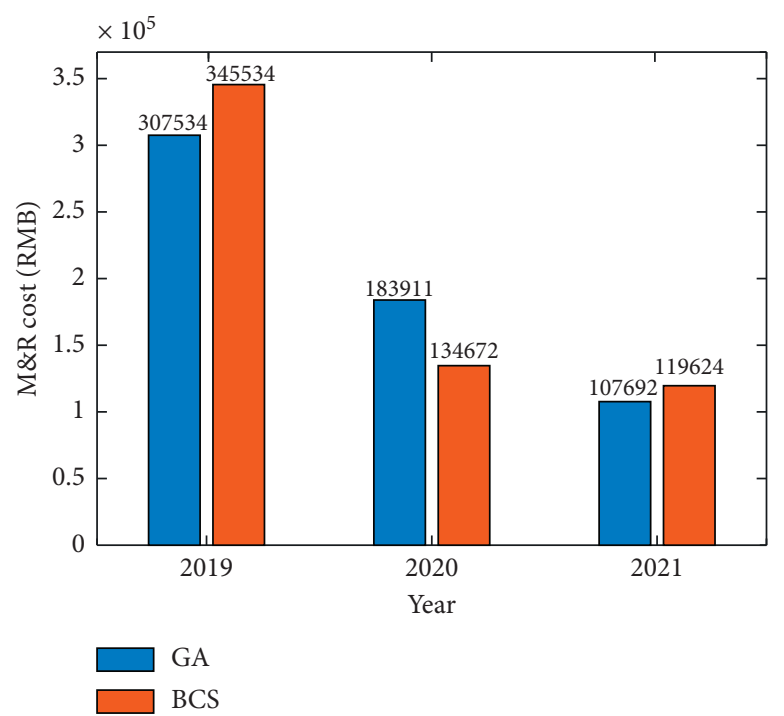

FIGURE 11: Budget allocation in each year under performance maximization.

$$
x^{t+1}=x^{t}+\alpha \varepsilon_{t}
$$

When $\varepsilon_{t}$ obeys a uniform distribution, equation (7) is a local random search; when $\varepsilon_{t}$ is drawn from a Lévy distribution, the step of the move may be larger, and equation (7) becomes a global random search. Therefore, the superior performance of BCS is due to its ability to perform both local and global searches simultaneously. 


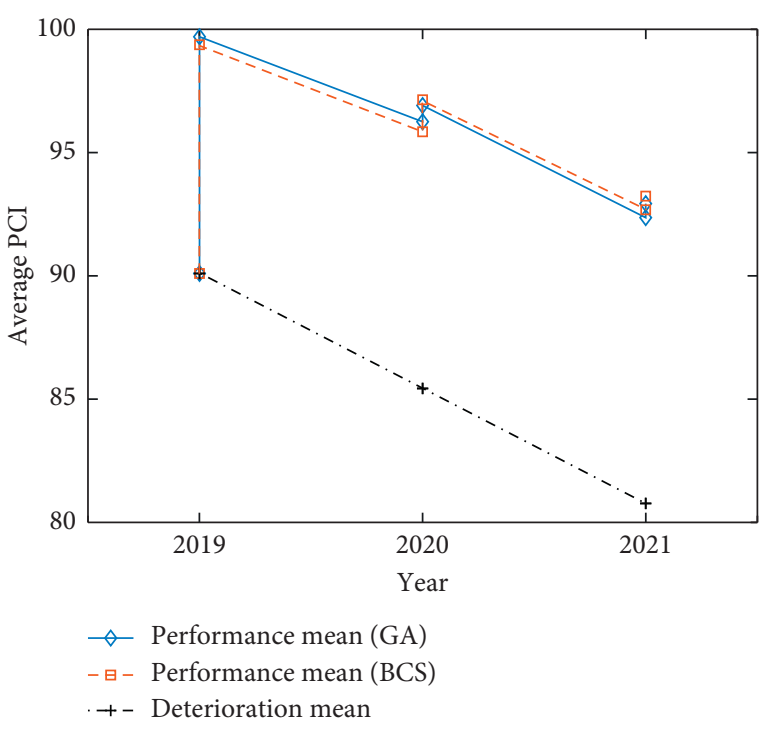

FIgURE 12: Pavement performance under biobjective model.

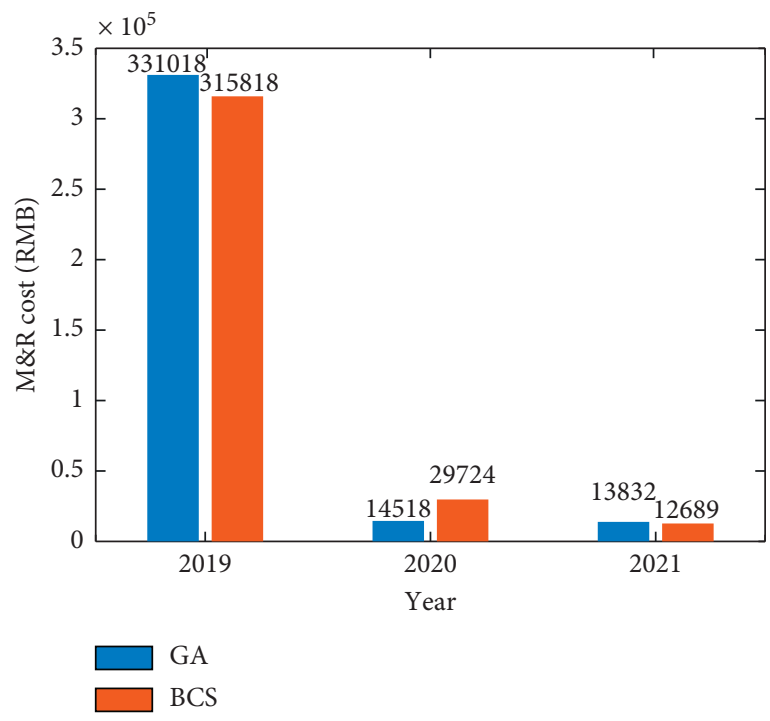

FIGURE 13: Budget allocation in each year under biobjective model.

\section{Summary and Conclusion}

In this study, the BCS algorithm and GA were performed to solve the combinatorial optimization problems in a multiyear pavement maintenance program using three typical hypothetical cases. The pavement performance degradation model used in these cases has taken uncertainty into account, and this makes the examples more representative. The results in all these cases show that BCS is not only suitable for combinatorial optimization models in pavement maintenance management but also better than GA in alleviating premature convergence. The advantage of BCS is particularly important for large-scale optimization problems in pavement maintenance management. With such an effective algorithm, the decision maker could make appropriate trade-off decisions.
The major study limitation is that the examples used are based on certain assumptions about performance prediction and decision tree. The reason for this phenomenon is that maintenance work in China is still at the initial stage and lacks mature performance prediction models and decision trees. Compared with GA, although BCS could converge to a better solution, BCS has an extra binary processing step, which leads to a slight increase in calculation time. In future research, BCS and other popular algorithms such as the PSO may be hybridized to produce better results. The initial feasible solutions are not generated randomly but through a heuristic algorithm, which may achieve better results. Although the BCS presented here is benchmarked in solving the combinatorial optimization of the pavement maintenance management problem, it could be generalizable to a wide range of problems regarding the management of different infrastructure assets.

\section{Data Availability}

The pavement data used to support the findings of this study are provided in Supplementary Materials. The MATLAB codes are provided in Supplementary Materials.

\section{Conflicts of Interest}

The authors declare that there are no conflicts of interest regarding the publication of this paper.

\section{Acknowledgments}

Thanks are due to Shanxi Department of Transportation for providing basic research data and financial support. This work was supported by the Science and Technology Projects of Shanxi Provincial Department of Transportation (Project nos. 7921000089, 8521002647, and 2018-1-32).

\section{Supplementary Materials}

Supplementary materials include Tables 4-9 and six MATLAB codes (see the file of "MATLAB code of six cases.docx"). The codes are provided to facilitate readers to reproduce the results of this study. A binary cuckoo search (BCS) algorithm and a genetic algorithm (GA) are used to calculate the results of the three cases in this study, so there are six corresponding MATLAB codes. In the fourth line of each MATLAB code, the file of "Data for this paper.xlsx" is the content of Table 9 in Supplementary Materials, which is the original data. In the file of "MATLAB codes of six cases.docx," the six MATLAB codes are explained as follows: for the first case with BCS, it aims to minimize total maintenance and rehabilitation (M\&R) costs, and it is solved by the BCS algorithm; for the first case with GA, it aims to minimize total M\&R costs, and it is solved by the GA. For the second case with BCS, it aims to maximize pavement performance, and it is solved by the BCS algorithm; for the second case with GA, it aims to maximize pavement performance, and it is solved by the GA. For the third case with $\mathrm{BCS}$, it aims to minimize total M\&R costs and maximize performance simultaneously, and it is solved by the BCS 
algorithm; for the third case with GA, it aims to minimize total $M \& R$ costs and maximize performance simultaneously, and it is solved by the GA. (Supplementary Materials)

\section{References}

[1] L. Chen and Q. Bai, "Optimization in decision making in infrastructure asset management: a review," Applied Sciences, vol. 9, no. 7, pp. 1380-7, 2019.

[2] J. Santos, A. Ferreira, and G. Flintsch, "An adaptive hybrid genetic algorithm for pavement management," International Journal of Pavement Engineering, vol. 20, no. 3, pp. 266-286, 2019.

[3] C. Blum and A. Roli, "Metaheuristics in combinatorial optimization," ACM Computing Surveys, vol. 35, no. 3, pp. 268-308, 2003.

[4] N. R. Tayebi, F. M. Nejad, and M. Mola, "Comparison between GA and PSO in analyzing pavement management activities," Journal of Transportation Engineering, vol. 140, no. 1, pp. 99-104, 2014.

[5] V. Yepes, C. Torres-Machi, A. Chamorro, and E. Pellicer, "Optimal pavement maintenance programs based on a hybrid greedy randomized adaptive search procedure algorithm," Journal of Civil Engineering and Management, vol. 22, no. 4, pp. 540-550, 2016.

[6] Z. Li and K. C. Sinha, "Methodology for multicriteria decision making in highway asset management," Transportation Research Record: Journal of the Transportation Research Board, vol. 1885, no. 1, pp. 79-87, 2004.

[7] J. C. Chu and K.-H. Huang, "Mathematical programming framework for modeling and comparing network-level pavement maintenance strategies," Transportation Research Part B: Methodological, vol. 109, pp. 1-25, 2018.

[8] J. H. Holland, Adaptation in Natural and Artificial Systems, University of Michigan press, Ann Arbor, MI, USA, 1975.

[9] Z.-D. Xu, X.-H. Huang, F.-H. Xu, and J. Yuan, "Parameters optimization of vibration isolation and mitigation system for precision platforms using non-dominated sorting genetic algorithm," Mechanical Systems and Signal Processing, vol. 128, pp. 191-201, 2019.

[10] A. Ardakani and A. Kordnaeij, "Soil compaction parameters prediction using GMDH-type neural network and genetic algorithm," European Journal of Environmental and Civil Engineering, vol. 23, no. 4, pp. 449-462, 2019.

[11] J. Reynolds, M. W. Ahmad, Y. Rezgui, and J.-L. Hippolyte, "Operational supply and demand optimisation of a multivector district energy system using artificial neural networks and a genetic algorithm," Applied Energy, vol. 235, pp. 699713, 2019.

[12] W. T. Chan, T. F. Fwa, and C. Y. Tan, "Road-maintenance planning using genetic algorithms. I: Formulation," Journal of Transportation Engineering, vol. 120, no. 5, 1994.

[13] T. F. Fwa, W. T. Chan, and C. Y. Tan, "Genetic-algorithm programming of road maintenance and rehabilitation," Journal of Transportation Engineering, vol. 122, no. 3, pp. 246-253, 1996.

[14] J. Lee and S. Madanat, "A joint bottom-up solution methodology for system-level pavement rehabilitation and reconstruction," Transportation Research Part B: Methodological, vol. 78, pp. 106-122, 2015.

[15] T. F. Fwa, W. T. Chan, and K. Z. Hoque, "Multiobjective optimization for pavement maintenance programming," Journal of Transportation Engineering, vol. 126, no. 5, pp. 367-374, 2000.
[16] B. Yu, X. Gu, F. Ni, and R. Guo, "Multi-objective optimization for asphalt pavement maintenance plans at project level: integrating performance, cost and environment," Transportation Research Part D: Transport and Environment, vol. 41, pp. 64-74, 2015.

[17] J. Santos, A. Ferreira, and G. Flintsch, "A multi-objective optimization-based pavement management decision-support system for enhancing pavement sustainability," Journal of Cleaner Production, vol. 164, pp. 1380-1393, 2017.

[18] A. Khavandi Khiavi and H. Mohammadi, "Multiobjective optimization in pavement management system using NSGAII method," Journal of Transportation Engineering, Part B: Pavements, vol. 144, no. 2, Article ID 04018016, 2018.

[19] K. C. P. Wang, V. Nguyen, and J. P. Zaniewski, "Genetic algorithms-based network optimization system with multiple objectives," Transportation Research Record: Journal of the Transportation Research Board, vol. 2016, no. 1, pp. 85-96, 2007.

[20] W. Sindi and B. Agbelie, "Assignments of pavement treatment options: genetic algorithms versus mixed-integer programming," Journal of Transportation Engineering, Part B: Pavements, vol. 146, no. 2, Article ID 04020008, 2020.

[21] G. Abu-Lebdeh, H. Chen, and M. Ghanim, "Improving performance of genetic algorithms for transportation systems: case of parallel genetic algorithms," Journal of Infrastructure Systems.vol. 22, no. 4, pp. 1-8, 2016.

[22] X.-S. Yang and S. Suash Deb, "Cuckoo search via Lévy flights," in Proceedings of the 2009 World Congress on Nature \& Biologically Inspired Computing (NaBIC), pp. 210-214, Coimbatore, India, December 2009.

[23] X. S. Yang and S. Deb, "Engineering optimisation by cuckoo search," International Journal of Mathematical Modelling and Numerical Optimisation, vol. 1, no. 4, p. 330, 2010.

[24] Y. S. M. Wang, X. S. He, and Y. Wang, "Markov model and convergence analysis based on cuckoo search algorithm," Computer Engineering, vol. 38, no. 11, pp. 180-182, 2012.

[25] A. Gherboudj, A. Layeb, and S. Chikhi, "Solving 0-1 knapsack problems by a discrete binary version of cuckoo search algorithm," International Journal of Bio-Inspired Computation, vol. 4, no. 4, p. 229, 2012.

[26] D. Rodrigues, "BCS: a binary cuckoo search algorithm for feature selection," in Proceedings of the 2013 IEEE International Symposium on Circuits and Systems (ISCAS2013), Beijing, China, May 2013.

[27] K. K. Bhattacharjee and S. P. Sarmah, "A binary cuckoo search algorithm for knapsack problems," in Proceedings of the IEOM 2015-5th International Conference on Industrial Engineering and Operations Management, pp. 1-5, Dubai, United Arab Emirates, March 2015.

[28] J. García, F. Altimiras, A. Peña, G. Astorga, and O. Peredo, “A binary cuckoo search big data algorithm applied to large-scale crew scheduling problems," Complexity, vol. 2018, Article ID 8395193, , 2018.

[29] N. V. Phanendra Babu and P. Suresh Babu, "A robust widearea measurement system for southern region of indian power grid using binary cuckoo search (BCS): a case study," Journal of Electrical Systems, vol. 12, no. 1, pp. 45-67, 2016.

[30] P. Yuli, Ed., Highway Performance Assessment Standards (JTG 5210-2018), Ministry of Transport of the People's Republic of China, Beijing, China, 2018.

[31] L. Yao, Q. Dong, F. Ni, J. Jiang, X. Lu, and Y. Du, "Effectiveness and cost-effectiveness evaluation of pavement treatments using life-cycle cost analysis," Journal of Transportation Engineering, Part B: Pavements, vol. 145, no. 2, Article ID 04019006, 2019. 
[32] R. N. Mantegna, "Fast, accurate algorithm for numerical simulation of Lévy stable stochastic processes," Physical Review E, vol. 49, no. 5, p. 4677, 1994.

[33] X. Yang, Nature-Inspired Metaheuristic Algorithms, Luniver Press, Bristol, UK, 2010.

[34] A. Elkeran, "A new approach for sheet nesting problem using guided cuckoo search and pairwise clustering," European Journal of Operational Research, vol. 231, no. 3, pp. 757-769, 2013.

[35] M. Shuaizhi, Research on Optimized Allocation on Pavement Maintenance Fund, Southeast University, Nanjing, China, 2019. 